\title{
Prediction of Chloride Diffusion in Concrete Structure Using Meshless Methods
}

\author{
Ling Yao, ${ }^{1}$ Xiaolu Li, ${ }^{1}$ Ling Zhang, ${ }^{1}$ and Lingling Zhang ${ }^{2}$ \\ ${ }^{1}$ State Key Laboratory for Strength and Vibration of Mechanical Structures, Xian Jiaotong University, Xian 710049, China \\ ${ }^{2}$ School of Human Settlements and Civil Engineering, Xian Jiaotong University, Xian 710049, China
}

Correspondence should be addressed to Ling Zhang; zhangl@mail.xjtu.edu.cn

Received 29 June 2016; Revised 10 October 2016; Accepted 16 November 2016

Academic Editor: Luciano Lamberti

Copyright (C) 2016 Ling Yao et al. This is an open access article distributed under the Creative Commons Attribution License, which permits unrestricted use, distribution, and reproduction in any medium, provided the original work is properly cited.

Degradation of RC structures due to chloride penetration followed by reinforcement corrosion is a serious problem in civil engineering. The numerical simulation methods at present mainly involve finite element methods (FEM), which are based on mesh generation. In this study, element-free Galerkin (EFG) and meshless weighted least squares (MWLS) methods are used to solve the problem of simulation of chloride diffusion in concrete. The range of a scaling parameter is presented using numerical examples based on meshless methods. One- and two-dimensional numerical examples validated the effectiveness and accuracy of the two meshless methods by comparing results obtained by MWLS with results computed by EFG and FEM and results calculated by an analytical method. A good agreement is obtained among MWLS and EFG numerical simulations and the experimental data obtained from an existing marine concrete structure. These results indicate that MWLS and EFG are reliable meshless methods that can be used for the prediction of chloride ingress in concrete structures.

\section{Introduction}

Reinforced concrete (RC) structures form the basis for most construction in civil engineering. However, a considerable number of reinforced concrete structures cannot achieve its design service life because of premature durability problems. Many factors influence the durability of a structure, including chloride ingress, carbonation resulting from penetrating carbon dioxide, and moisture transport. Extensive research has shown that chloride ingress in concrete is one of the most significant processes that can seriously impair the long term durability of RC structures [1-3]. Conventional testing of chloride penetration into concrete is time-consuming, making it advantageous to numerically simulate chloride ingress into concrete.

Many studies have focused on Fick's second law of diffusion as the basis for the description of chloride transport in concrete, assuming that diffusion is the dominant transport mechanism. However, obtaining a sound analytical solution can be difficult in practical engineering of complicated structures. Therefore, development of more effective methods for predicting chloride concentration in concrete structures is necessary. Many researchers have proposed numerical simulation methods to describe the phenomenon of chloride transport in concrete [4-6]. In general, these methods are based on the finite element methods (FEM), the finite difference methods (FDM), or similar approaches. However, these methods are limited in terms of overcoming problems, such as complex pretreatment and mesh generation. In contrast, meshless methods only employ data at the nodes and hence do not require that the solution domain be subdivided into many smaller regions. Consequently, meshless methods have a simple preprocessing step and offer high accuracy for scientific and engineering problems.

Recently, many meshless methods have been proposed in literature [7-14], including the smoothed particle hydrodynamic method, the diffuse element method, the EFG method, the reproducing kernel particle method, the finite point method, and the meshless local Petrov-Galerkin method as well as many others. The point collocation methods and the Galerkin method of discretization are among the most commonly used meshless methods. The Galerkin method is a famous example of a meshless method, which was proposed by Belytschko et al. in 1994 [7]. The EFG method is a type 
of Galerkin meshless method. The other type of a widely used meshless method is the point collocation method. The meshless weighted least squares (MWLS) method is a type of point collocation methods. The MWLS and EFG methods have been successfully applied to solve problems regarding conduction of heat transfer [15]. However, few studies have reported on the application of meshless methods to solve the problems of chloride transport in concrete. In reality a variety of transport mechanisms occur, but chloride transport in concrete is modelled as a diffusion process in current research. Bitaraf and Mohammadi in [15] reported a FPM developed and adopted for solving the chloride diffusion equation in concrete for prediction of service life of concrete structures and initiation time of corrosion of reinforcements. Guo et al. in [16] reported the use of the transient meshless boundary element method for predicting chloride diffusion, which emphasized time-dependent nonlinear coefficients.

In the study reported in this paper, the MWLS and EFG methods were used to solve problems of chloride transport by diffusion in concrete structure. This paper is organized as follows. Section 2 presents a brief introduction of the moving least squares (MLS) approximations. The implementation of EFG and MWLS methods is shown in Sections 3 and 4. The numerical examples are demonstrated and the results compared with other methods in Section 5. Finally, Section 6 presents the concluding remarks.

\section{MLS Approximation Scheme}

MLS approximation is a well-known meshless interpolation scheme. MLS is adopted as an approximation scheme in MWLS and EFG methods. In MLS approximation, the function $u(x)$ is approximated by $u^{h}(x)$ as follows:

$$
u(x) \approx u^{h}(x)=\sum_{i=1}^{m} p_{i}(x) a_{i}(x)=\mathbf{p}^{\mathrm{T}}(\mathbf{x}) \mathbf{a}(\mathbf{x}),
$$

where $\mathbf{p}^{\mathbf{T}}(\mathbf{x})=\left[p_{1}(x), p_{2}(x), \ldots, p_{m}(x)\right]^{T}$ is a complete polynomial basis of $m$ order, $\mathbf{a}(\mathbf{x})$ is a vector containing coefficients, $\mathbf{a}(\mathbf{x})=\left[a_{1}(x), a_{2}(x), \ldots, a_{n}(x)\right]^{T}$, which are functions of the coordinates $\mathbf{x}$. For a linear basis $m=3$, $\mathbf{p}^{\mathrm{T}}(\mathbf{x})=\mathbf{p}^{\mathrm{T}}(x, y)=[1, x, y]$ and $\mathbf{p}^{\mathrm{T}}(\mathbf{x})=\mathbf{p}^{\mathrm{T}}(x, y)=$ $\left[1, x, y, x y, x^{2}, y^{2}\right]$ whereas for a quadratic basis $m=6$. $J$ is a function of the weighted residual, which represents the approximated values of the field function at the nodes, $u_{I}=$ $u\left(x_{I}\right)$, and

$$
\begin{aligned}
J & =\sum_{I=1}^{N} w_{I}(x)\left[u^{h}\left(x, x_{I}\right)-u\left(x_{I}\right)\right]^{2} \\
& =\sum_{I=1}^{N} w_{I}(x)\left[\sum_{i=1}^{m} \mathbf{p}^{\mathbf{T}}\left(x_{I}\right) \mathbf{a}(\mathbf{x})-u_{I}\right]^{2} .
\end{aligned}
$$

$w_{I}(x)=w\left(x-x_{I}\right)$, which is a weight function, and $u_{I}$ is the nodal parameter of the field variable at node $I$ in which the coefficient $\mathbf{a}(\mathbf{x})$ can be chosen to minimize the weighted residual.

$$
\begin{aligned}
& \mathbf{A}(\mathbf{x})=\sum_{I=1}^{N} W_{I}(x) p\left(x_{I}\right) p^{T}\left(x_{I}\right), \\
& \mathbf{B}(\mathbf{x}) \\
& \quad=\left[w_{1}(x) p\left(x_{1}\right) w_{2}(x) p\left(x_{2}\right) \cdots w_{N}(x) p\left(x_{N}\right)\right], \\
& \mathbf{a}(\mathbf{x})=\mathbf{A}^{-1}(\mathbf{x}) \mathbf{B}(\mathbf{x}) \mathbf{u}, \\
& \mathbf{u}=\left[u_{1}, u_{2}, \ldots, u_{n}\right]^{T} .
\end{aligned}
$$

Substituting (5) into (1) yields

$$
u^{h}(x)=\sum_{I=1}^{N} N_{I}(x) u_{I}=\mathbf{p}^{\mathbf{T}}(\mathbf{x}) \mathbf{A}^{-1}(\mathbf{x}) \mathbf{B}(\mathbf{x}) \mathbf{u},
$$

where $\mathbf{N}(\mathbf{x})$ is a shape function.

$$
\mathbf{N}(\mathbf{x})=\mathbf{p}^{\mathrm{T}}(\mathbf{x}) \mathbf{A}^{-1}(\mathbf{x}) \mathbf{B}(\mathbf{x})=\mathbf{r}^{\mathrm{T}}(\mathbf{x}) \cdot \mathbf{B}(\mathbf{x}) .
$$

In the MLS approximation, the continuity relates not only to the basic function but also to the weight function. The weight function plays various important roles, the first of which is to provide weighting of the residuals at different nodes in the support domain. The second role is to ensure that the nodes leave or enter the support domain in a gradual (smooth) manner when $x$ moves, thereby ensuring the compatibility condition [17]. In this study, the weight function is selected as follows.

The cubic spline weight function is defined by

$$
\begin{aligned}
w\left(x-x_{I}\right) & =w(r) \\
& = \begin{cases}\frac{2}{3}-4 r^{2}+4 r^{3} & r \leq \frac{1}{2} \\
\frac{4}{3}-4 r+4 r^{2}-\frac{4}{3} r^{3} & \frac{1}{2}<r \leq 1 \\
0 & r>1,\end{cases}
\end{aligned}
$$

the quartic spline weight function is defined by

$$
w\left(x-x_{I}\right)=w(r)= \begin{cases}1-6 r^{2}+8 r^{3}-3 r^{4} & r \leq 1 \\ 0 & r>1\end{cases}
$$

and the normalized Gaussian weight function is given through

$$
w(r)= \begin{cases}\frac{e^{-r^{2} \beta^{2}}-e^{-\beta^{2}}}{1-e^{-\beta^{2}}} & r \leq 1 \\ 0 & r>1\end{cases}
$$


where

$$
\begin{aligned}
r & =\frac{d_{I}}{d_{m_{I}}}, \\
\left(r_{x}\right)_{I} & =\frac{\left\|x-x_{I}\right\|}{d_{m x_{I}}}, \\
\left(r_{y}\right)_{I} & =\frac{\left\|y-y_{I}\right\|}{d_{m y_{I}}}, \\
d_{I} & =\left\|x-x_{I}\right\|, d_{m x_{I}}=d_{\max } c_{x_{I}}, d_{m y_{I}}=d_{\max } c_{y_{I}},
\end{aligned}
$$

and $d_{\max }$ represents the dimensionless scaling parameter and $c_{x_{I}}, c_{y_{I}}$ are the distances to the nearest neighbors at $I$ node.

\section{EFG Method for Chloride Diffusion in Concrete}

The distribution of chloride in the problem domain $\Omega$ is governed by Fick's second law:

$$
\frac{\partial C}{\partial t}=\frac{\partial}{\partial x}\left(D_{x} \frac{\partial C}{\partial x}\right)+\frac{\partial}{\partial y}\left(D_{y} \frac{\partial C}{\partial y}\right) \quad \text { in } \Omega
$$

where $C=C(x, y, t)$ represents concentration, $t$ is time, and $D_{x}, D_{y}$ represent the diffusion coefficients. In this article, assuming $D$ is the same in $x$ and $y$ directions, the initial concentration of chloride ion present in concrete is

$$
C(x, y, 0)=0 \quad \text { in } \Omega .
$$

Dirichlet's boundary condition is given by

$$
C=\bar{C}, \quad \text { on } \Gamma_{1} \text {. }
$$

The weak form of equation (13) is expressed as

$$
\int_{\Omega} \nabla w\left(D_{x} \frac{\partial C}{\partial x}+D_{y} \frac{\partial C}{\partial y}\right) d \Omega-\int_{\Omega} w\left(\frac{\partial C}{\partial t}\right) d \Omega=0
$$

The function $\Pi(C)$ can be written as

$$
\begin{aligned}
\Pi(C)= & \frac{1}{2} \int_{\Omega}\left[D_{x}\left(\frac{\partial C}{\partial x}\right)^{2}+D_{y}\left(\frac{\partial C}{\partial y}\right)^{2}\right] d \Omega \\
& +\int_{\Omega} C\left(\frac{\partial C}{\partial t}\right) d \Omega .
\end{aligned}
$$

Let $\delta \Pi=0$, and then

$$
\begin{aligned}
\int_{\Omega} \delta C & \frac{\partial C}{\partial t} d \Omega+\int_{\Omega} \delta(L C)^{T} D(L C) d \Omega=0, \\
L(\bullet) & =\left[\begin{array}{c}
\frac{\partial}{\partial x} \\
\frac{\partial}{\partial y}
\end{array}\right](\bullet), \\
D & =\left[\begin{array}{cc}
D_{x} & 0 \\
0 & D_{y}
\end{array}\right], \\
\frac{\partial C}{\partial t} & =\frac{\partial}{\partial t} \sum_{I=1}^{n} N_{I}(x) C_{I}(t)=\sum_{I=1}^{n} N_{I}(x) \frac{\partial C_{I}(t)}{\partial t} \\
& =N_{\mathbf{I}}(\mathbf{x}) \dot{\mathbf{C}}, \\
\dot{C} & =\left(\frac{\partial C_{1}(t)}{\partial t}, \frac{\partial C_{2}(t)}{\partial t}, \ldots, \frac{\partial C_{n}(t)}{\partial t}\right)^{T}, \\
L C & \left.=L \sum_{I=1}^{n} N_{I}(x) C(t)=\sum_{I=1}^{n}\left[\frac{\partial}{\partial x}\right] \frac{\partial}{\partial y}\right] N_{I}(x) C_{I}(t) \\
& =\mathbf{E}(\mathbf{x}) \mathbf{C},
\end{aligned}
$$

where $\mathbf{E}$ is

$$
\begin{aligned}
\mathbf{E}(\mathbf{x}) & =\left(\mathbf{E}_{1}(\mathbf{x}), \mathbf{E}_{2}(\mathbf{x}), \ldots, \mathbf{E}_{\mathbf{n}}(\mathbf{x})\right), \\
\mathbf{E}_{\mathbf{I}}(\mathbf{x}) & =\left[\begin{array}{l}
\mathbf{N}_{\mathbf{I}, \mathbf{x}}(\mathbf{x}) \\
\mathbf{N}_{\mathbf{I}, \mathbf{y}}(\mathbf{x})
\end{array}\right] .
\end{aligned}
$$

Lagrange's Multiplier technique was used to impose the essential boundary conditions in the EFG method; hence, substituting (20), (21), and (22) into (16) we obtain

$$
\begin{aligned}
\int_{\Omega} \delta & {[N(x) C][N(x) \dot{C}] d \Omega } \\
& +\int_{\Omega} \delta[E(x) C]^{T} \widetilde{D}[E(x) C] d \Omega \\
& +\int_{\Gamma_{1}} \delta \lambda^{T}(C-\bar{C}) d \Gamma+\int_{\Gamma_{1}} \delta C^{T} \lambda d \Gamma=\mathbf{0} .
\end{aligned}
$$

Finally, (24) can be written as

$$
\begin{array}{r}
{[M]\{\dot{C}\}+[K]\{C\}+[G]\{\Lambda\}=0,} \\
{\left[G^{T}\right]\{C\}-\{Q\}=0,}
\end{array}
$$


where

$$
\begin{aligned}
{[M] } & =\int_{\Omega} N_{I}(x) N_{J}(x) d \Omega, \\
{[K] } & =\int_{\Omega}\left[D_{x} \frac{\partial N_{I}}{\partial x} \frac{\partial N_{J}}{\partial x}+D_{y} \frac{\partial N_{I}}{\partial y} \frac{\partial N_{J}}{\partial y}\right] d \Omega, \\
{[G] } & =\int_{\Gamma_{1}} N_{\lambda}^{T} N d \Gamma, \\
{[Q] } & =\int_{\Gamma_{1}} N_{\lambda}^{T} \bar{C} d \Gamma .
\end{aligned}
$$

The time interval $[0, t]$ is subdivided into a finite number of equal subintervals $\Delta t$. By using the $\theta$ method, (25) can be written as

$$
\begin{aligned}
\bar{K} C^{n+1}= & \bar{Q}^{n+1}, \\
\bar{K}= & \frac{[M]}{\Delta t}+\theta[K], \\
\bar{Q}^{n+1}= & \left(\frac{[M]}{\Delta t}+(\theta-1)[K]\right) C^{n}+(1-\theta) f^{n} \\
& +\theta f^{n+1} .
\end{aligned}
$$

Equation (25) can be written as

$$
\left[\begin{array}{cc}
\bar{K} & G \\
G^{T} & 0
\end{array}\right]\left[\begin{array}{c}
C^{k+1} \\
\Lambda
\end{array}\right]=\left[\begin{array}{l}
R \\
Q
\end{array}\right]
$$

with

$$
\begin{aligned}
\bar{K} & =[M]+\Delta t \theta[K], \\
R & =([M]-\Delta t(1-\theta)[K])\{C\}^{k},
\end{aligned}
$$

where $\theta=0.5, \theta=2 / 3$, and $\theta=1$ are the CrankNicolson form, Galerkin form, and backward difference form, respectively.

\section{MWLS Method for Chloride Diffusion in Concrete}

The essential concept of MWLS is that the method is a weighted residual method; that is, the weight function is residual and the function $J_{R}$ is obtained by summation of the squares of residuals.

$$
J_{R}=\int_{\Omega} \alpha R^{T} R d \Omega+\int_{\Gamma} \beta \bar{R}^{T} \bar{R} d \Gamma .
$$

$\alpha, \beta$ are the weight coefficients, which when minimized, produce

$$
\int_{\Omega} \alpha\left(\frac{\partial R}{\partial u_{j}}\right)^{T} R d \Omega+\int_{\Gamma} \beta\left(\frac{\partial \bar{R}}{\partial u_{j}}\right)^{T} \bar{R} d \Gamma=0,
$$

where $j=1,2, \ldots, n \cdot w_{j}=\alpha\left(\partial R / \partial u_{j}\right)$ and $\bar{w}_{j}=\beta\left(\partial \bar{R} / \partial u_{j}\right)$ are the test functions. A discrete form is adopted to avoid integration as follows:

$$
\begin{aligned}
\sum_{k=1}^{n_{0}} \alpha_{k}\left(\frac{\partial R\left(x_{k}\right)}{\partial u_{j}}\right)^{T} \bar{R}\left(x_{k}\right) & \\
& +\sum_{k=1}^{n_{1}} \beta_{k}\left(\frac{\partial \bar{R}\left(x_{k}\right)}{\partial u_{j}}\right)^{T} \bar{R}\left(x_{k}\right)=0,
\end{aligned}
$$

where $\alpha_{k}, \beta_{k}$ are the weighted coefficients, $x_{k}$ is the collection node, and $n_{0}$ and $n_{1}$ are the number of evaluation points needed to satisfy the governing equation and the boundary conditions, respectively. An approximation function of residuals is set with the MLS scheme. In the present study, the penalty function approach was used to impose the essential boundary conditions in MWLS.

The residual of (31) is set according to (32):

$$
\Pi=\int_{\Omega} R_{0}^{2}(x) d \Omega+\int_{\Gamma_{1}} \alpha_{1} R_{1}^{2}(x) d \Gamma,
$$

where $\alpha_{1}=\left(10^{5} \sim 10^{8}\right) \times(D / L)^{2}[18], \alpha_{1}$ is the penalty parameter required to apply the boundary condition, and $L$ is the characteristic length in this problem. By adopting the variational principle in (31) and using the discrete form to avoid integration, finally this results into

$$
\begin{aligned}
{[M] \dot{C}+[K] C=} & f, \\
{[M]=} & -\sum_{s=1}^{N} D\left[\nabla^{2} N_{I}\left(x_{s}\right)\right] \cdot\left[N_{J}\left(x_{s}\right)\right], \\
{[K]=} & \sum_{s=1}^{N}\left[D \nabla^{2} N_{I}\left(x_{s}\right)\right] \cdot\left[D \nabla^{2} N_{J}\left(x_{s}\right)\right] \\
& +\sum_{s=1}^{N} \alpha_{1} N_{I}\left(x_{s}\right) N_{J}\left(x_{s}\right), \\
f= & \sum_{s=1}^{N_{1}} \alpha_{1} N_{I}\left(x_{s}\right) \bar{C} .
\end{aligned}
$$

The above equations are the computation format of MWLS with respect to chloride diffusion in concrete. Equation (34) is solved by the approach in the EFG method.

\section{Numerical Examples}

In the previous sections, the efficiency of the methods has been verified by using one-dimensional (1D) and twodimensional (2D) numerical examples to demonstrate the applicability of the proposed method for quantifying chloride ion diffusion in concrete structures. In the current analysis, one Gauss point in the $1 \mathrm{D}$ problem and $4 \times 4$ Gauss points in the $2 \mathrm{D}$ problem were used to perform integration in the EFG method. MATLAB codes were developed to obtain the EFG and MWLS results, whereas the FEM results were obtained using the same four-noded brick elements in COMSOL Multiphysics 4.3 software. 
TABLE 1: RMS (\%) error of different weight functions at 31 nodes for exposure time $t=20$ years.

\begin{tabular}{|c|c|c|c|c|c|c|}
\hline \multirow[t]{2}{*}{$\theta$} & \multicolumn{2}{|c|}{ Quartic spline } & \multicolumn{2}{|c|}{ Cubic spline } & \multicolumn{2}{|c|}{$\begin{array}{c}\text { Gaussian function } \\
\qquad(\beta=4.0)\end{array}$} \\
\hline & MWLS & EFG & MWLS & EFG & MWLS & EFG \\
\hline$\theta=0.5$ & 0.1888 & 0.1300 & 0.1441 & 0.0622 & 0.1725 & 0.8256 \\
\hline$\theta=2 / 3$ & 0.1485 & 0.1626 & 0.1097 & 0.0877 & 0.1397 & 0.8219 \\
\hline$\theta=1$ & 0.1068 & 0.2518 & 0.1008 & 0.1854 & 0.1211 & 0.8944 \\
\hline
\end{tabular}

TABLE 2: Results of MWLS, EFG, and FEM and exact solutions at a few specific locations (31 nodes, $t=20$ years).

\begin{tabular}{lcccc}
\hline $\begin{array}{l}\text { Location }(\mathrm{m}) \\
x\end{array}$ & MWLS & \multicolumn{2}{c}{ Chloride concentration (\%) } & FEM \\
\hline 0.01 & 4.1125 & EFG & 4.1174 & 4.1129 \\
0.03 & 2.5014 & 2.1132 & 2.5110 & 2.5058 \\
0.05 & 1.3109 & 1.3134 & 1.3096 & 1.3112 \\
0.07 & 0.5889 & 0.5855 & 0.5800 & 0.5826 \\
0.09 & 0.2268 & 0.2207 & 0.2182 & 0.2180 \\
0.11 & 0.0754 & 0.0702 & 0.0690 & 0.0682 \\
0.13 & 0.0229 & 0.0196 & 0.0186 & 0.0178 \\
0.15 & 0.011 & 0.00087 & 0.007637 & 0.0039 \\
\hline
\end{tabular}

5.1. 1D Case Study. The first example is that of a concrete slab of $0.15 \mathrm{~m}$ thickness. The left boundary is permanently subjected to a constant chloride concentration of 5\% (by mass of $\mathrm{NaCl}$ ). The initial chloride concentration is $0, d_{\max }=$ 2.5 , and for a linear basis $m=3$. In the first example, the diffusion coefficient is assumed to be constant $D=$ $1.5768 \times 10^{-12} \mathrm{~m}^{2} / \mathrm{s}$ and in the second example to be a timedependent function $D(t)=D_{0}\left(t_{0} / t\right)^{m}$, where $D_{0}$ is the diffusion coefficient at some reference time $t_{0}$ and $m$ denotes a material constant [18]. For this situation, $D=1.5768 \times$ $10^{-12} \times(0.1 / t)^{0.1} \mathrm{~m}^{2} / \mathrm{s}$ have been chosen. For the purpose of convergence studies, the root-mean square (RMS) error is defined as $R=(1 / n) \sqrt{\sum_{I=1}^{n} C\left(x_{I}\right)-C^{*}\left(x_{I}\right)}$, where $n$ is the number of sample points, $C\left(x_{I}\right)$ is the calculation result with simulation, and $C^{*}\left(x_{I}\right)$ denotes the result obtained with the analytical solution.

5.1.1. Coefficient D Is a Constant. The analytical solution for the $1 \mathrm{D}$ diffusion of chloride ions in concrete is

$$
C(x, t)=C_{s}\left(1-\operatorname{erf}\left(\frac{x}{2 \sqrt{D t}}\right)\right),
$$

where $\operatorname{erf}(\cdot)$ is the error function and $C_{s}=5 \%$ is assumed. The slab is discretized into 31 nodes with an exposure time $t=$ 20 years to implement the meshless methods. Table 1 shows the numerical comparison of the RMS error for different weight functions and $\theta$ with MWLS and EFG methods. The results indicate that the observed minimum error occurs when the cubic spline weight function is used and $\theta=1$ using the MWLS method. Therefore, the cubic spline weight function was chosen with $\theta=1$ in the numerical simulation that follows with the MWLS method. When using the EFG method, the minimum error occurred when the cubic spline weight function and $\theta=0.5$ were adopted, and hence the cubic spline weight function and $\theta=0.5$ were used in the EFG method analysis.

Table 2 shows a comparison of the chloride concentration using the MWLS, EFG, and FEM methods as well as the exact solution at several specific locations among the 31 nodes for $t=20$ years. The same number of nodes was used in all methods. Table 2 shows that the results of the meshless methods are very close to those of the analytical solution, as reflected in the very small errors.

Figure 1 shows the variations of RMS error as a function of the number of nodes using MWLS and EFG methods at exposure time $t=20$ years. The results show that, in the MWLS method, the error was reduced as the number of nodes increased, whereas, in the EFG method, the error reached a minimum when the number of nodes is 51 . However, the variations in computational (CPU) time with the different number of nodes using MWLS and EFG methods yielded different results as shown in Figure 2. This figure shows that the CPU time for the EFG method was longer and increased with the increase in the number of nodes, whereas that of the MWLS was much shorter as the number of nodes increases.

Figure 3 shows the change in chloride concentration with depth for exposure times of 1, 10, and 20 years. In this figure, $\mathrm{ClM}, \mathrm{C} 1 \mathrm{E}$, and $\mathrm{Cla}$ denote the chloride concentrations from MWLS, EFG, and the analytical solution, respectively, after one-year exposure employing 31 nodes. The results of the MWLS and EFG simulations agree well with the analytical results. When $t=20$ years, the RMS error using the MWLS and EFG methods is $0.1008 \%$ and $0.0622 \%$, respectively.

5.1.2. Coefficient D Is a Time-Dependent Function. In the second example, when the diffusion coefficient $D$ is not a 


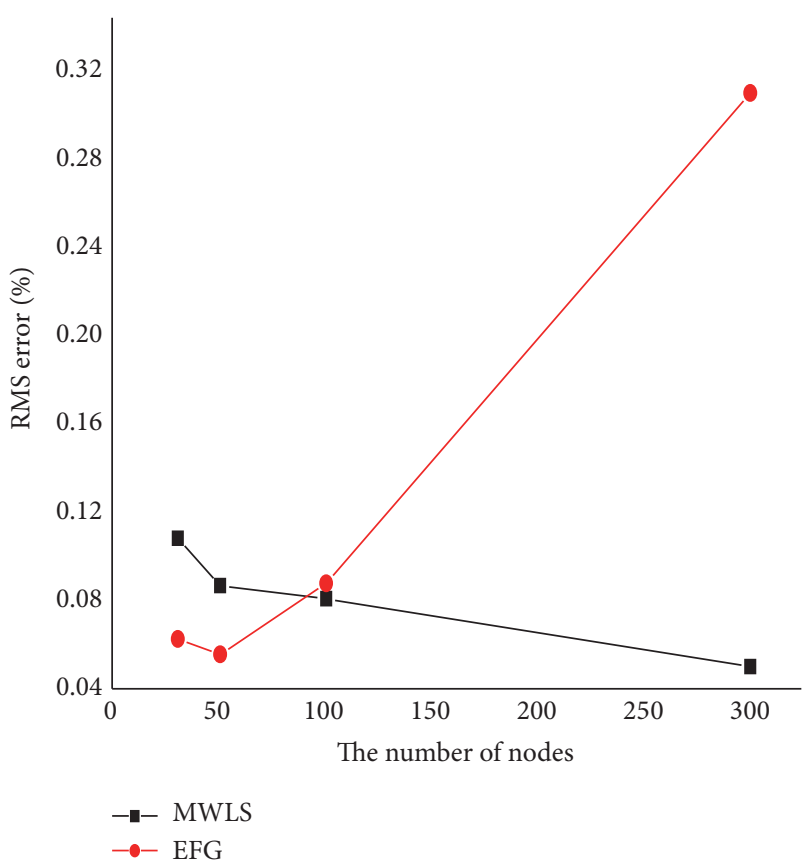

FIgURE 1: Variation of RMS error at different nodes.

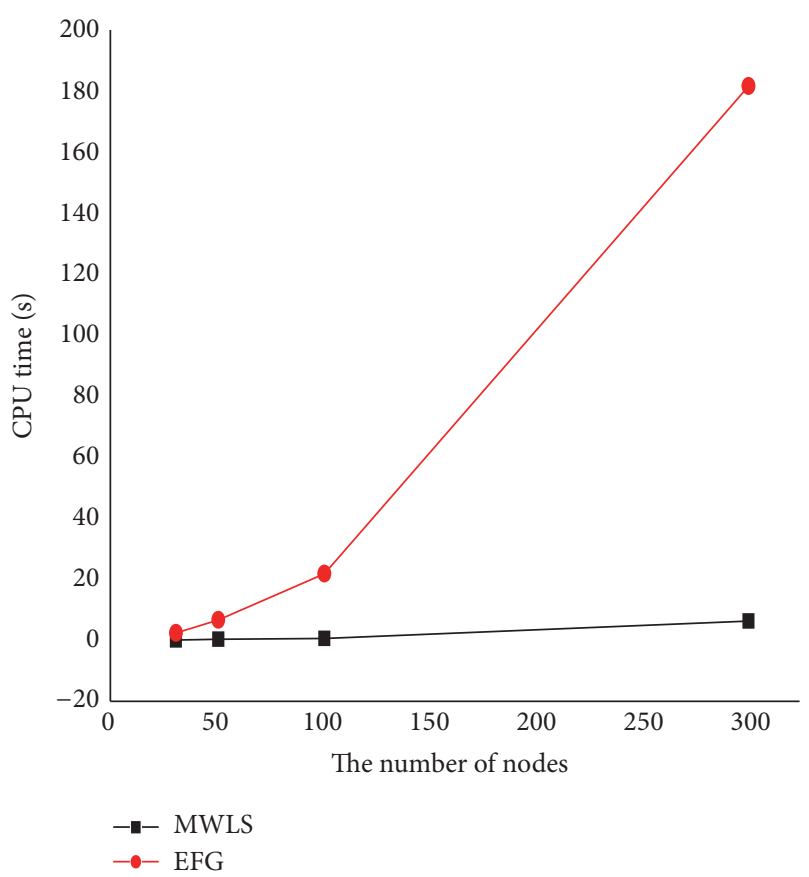

FIGURE 2: Variation of computational time with number of nodes.

constant but follows a time-dependent function, the analytical solution is represented by

$$
\frac{C}{C_{s}}=\left(1-\operatorname{erf}\left(\frac{x}{2 \sqrt{D_{a} \cdot t}}\right)\right)
$$

where

$$
D_{a}=\frac{D_{0}}{1-m}\left[\left(1+\frac{t_{0}}{t}\right)^{1-m}-\left(\frac{t_{0}}{t}\right)^{1-m}\right]\left(\frac{t_{0}}{t}\right)^{m} .
$$

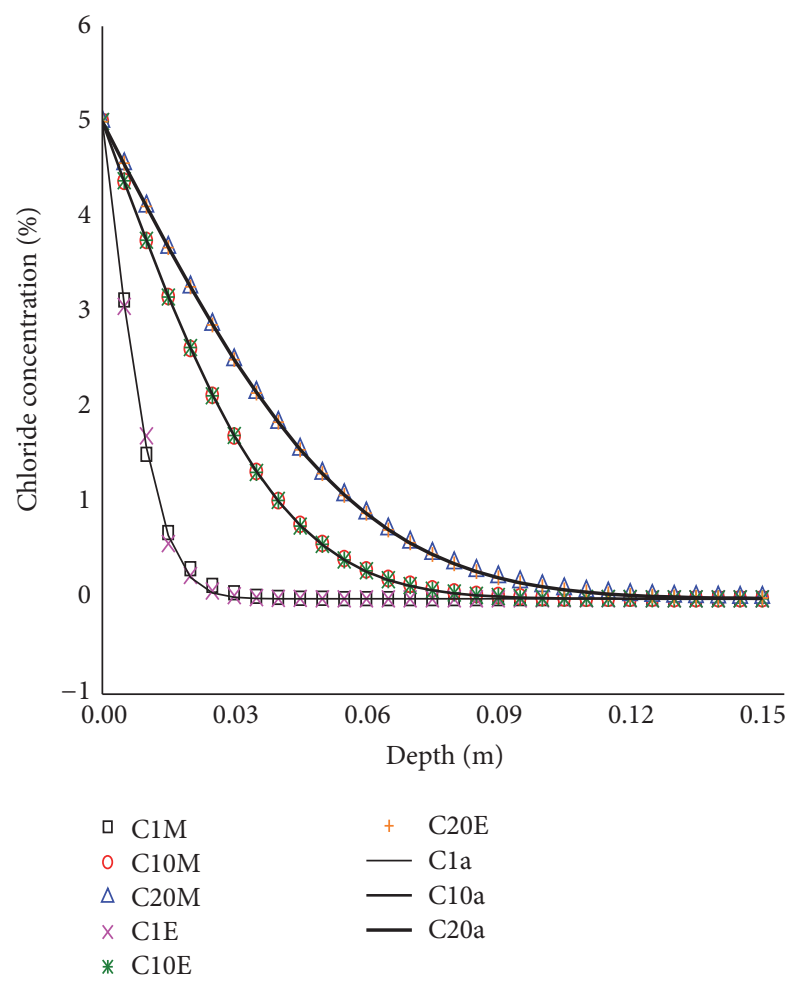

FIGURE 3: Variation of chloride concentration with depth.

TABLE 3: The initiation period of corrosion by different methods (at 31 nodes).

\begin{tabular}{lcccc}
\hline $\begin{array}{l}\text { Initiation period } \\
\text { (year) }\end{array}$ & MWLS & EFG & FEM & Exact solutions \\
\hline $\begin{array}{l}D \text { is a constant } \\
\begin{array}{l}\text { is a time-dependent } \\
\text { function }\end{array}\end{array}$ & 4.50 & 4.55 & 4.60 & 4.67 \\
\hline
\end{tabular}

The results shown in Figure 4 indicate a good agreement between results obtained with the MWLS and EFG methods and the analytical solution. RMS error was $1.4786 \%$ between the MWLS and the analytical results, whereas the RMS error of the EFG method was $0.0865 \%$ when $t=20$ years and 31 nodes were used. The initiation period ends at the start of reinforcement corrosion in RC structures which is assumed to occur when the critical chloride ion concentration $\left(C_{t}\right)$ is $0.1 \%$ at the level of the reinforcement, and here, at a depth of $5 \mathrm{~cm}$, it is obtained as shown in Table 3. The table clearly shows that predictions of the initiation periods of corrosion in different meshless methods are very close to the exact value as calculated with the analytical solution.

From these two examples, the MWLS and EFG methods appear to be effective in accurately predicting chloride concentrations in concrete structures. The accuracy of the EFG is higher than that of MWLS, but the error of MWLS remains small in actual engineering applications. 


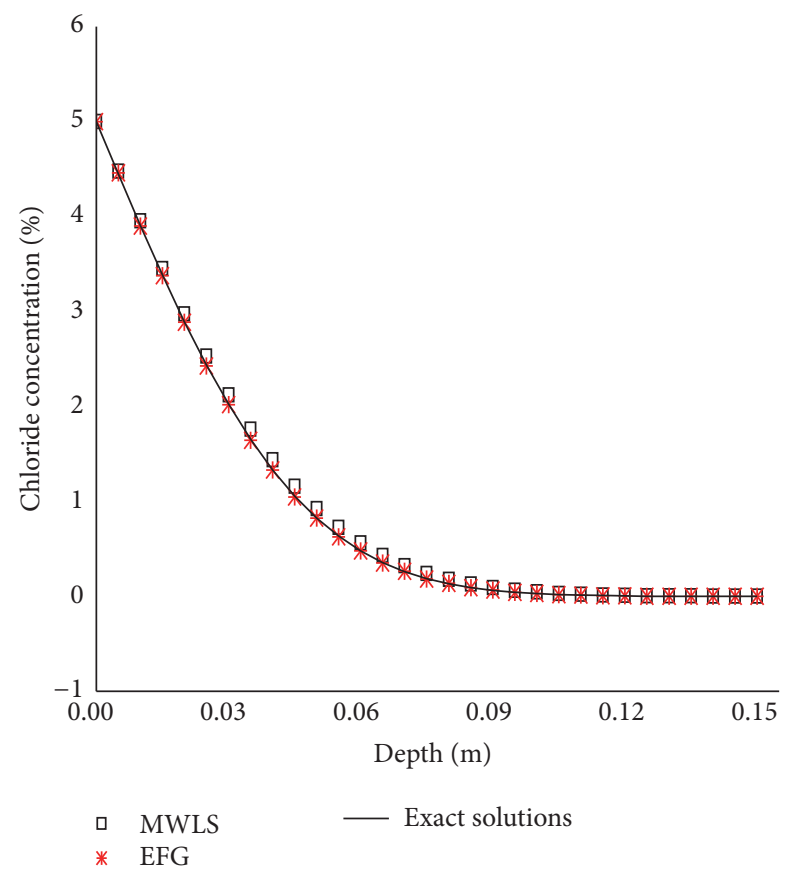

Figure 4: Chloride concentration-depth at time $=20$ years.

5.2. $2 D$ Problem. The rate of chloride diffusion in an actual reinforced concrete structure is very slow, and thus measuring long term diffusion is a slow, time-consuming process. In addition, few research studies have dealt with chloride diffusion over long periods. In this section, a concrete plate of $0.15 \mathrm{~m} \times 0.15 \mathrm{~m}$ was used as an example, as shown as Figure 5 . The left and bottom boundaries of the plate were subjected to a chloride concentration of $0.6 \%$ (by mass of concrete) as Dirichlet's boundaries and the right and top boundaries were isolated. The initial chloride concentration was 0 , which is similar to that reported by Guo et al. in [16].

5.2.1. Coefficient D Is a Constant. A regular distribution of $11 \times 11$ nodes was selected in $\Omega$ domain for all numerical methods. In the computing process, the scaling factor of the support was 2.25, quadratic basis was $m=6$ in the MWLS, and linear basis was $m=3$ in the EFG. The 2D characteristic of the example shows that when the diffusion coefficient $D$ is a constant $\left(D=9.38 \times 10^{-12} \mathrm{~m}^{2} / \mathrm{s}\right)$, the analytical solution is as follows [16]:

$$
\begin{aligned}
& C(x, y, t) \\
& \quad=C_{s}\left[1-\operatorname{erf}\left(\frac{x}{2 \sqrt{D_{x} t}}\right) \operatorname{erf}\left(\frac{y}{2 \sqrt{D_{y} t}}\right)\right] .
\end{aligned}
$$

Table 4 provides a comparison of the results obtained using the MWLS and those obtained by EFG and FEM, as well as the analytical results according to (41) at several specific sample locations. The MWLS and EFG results were basically the same as the exact solutions. The error of MWLS remains within a permissible range in practice.

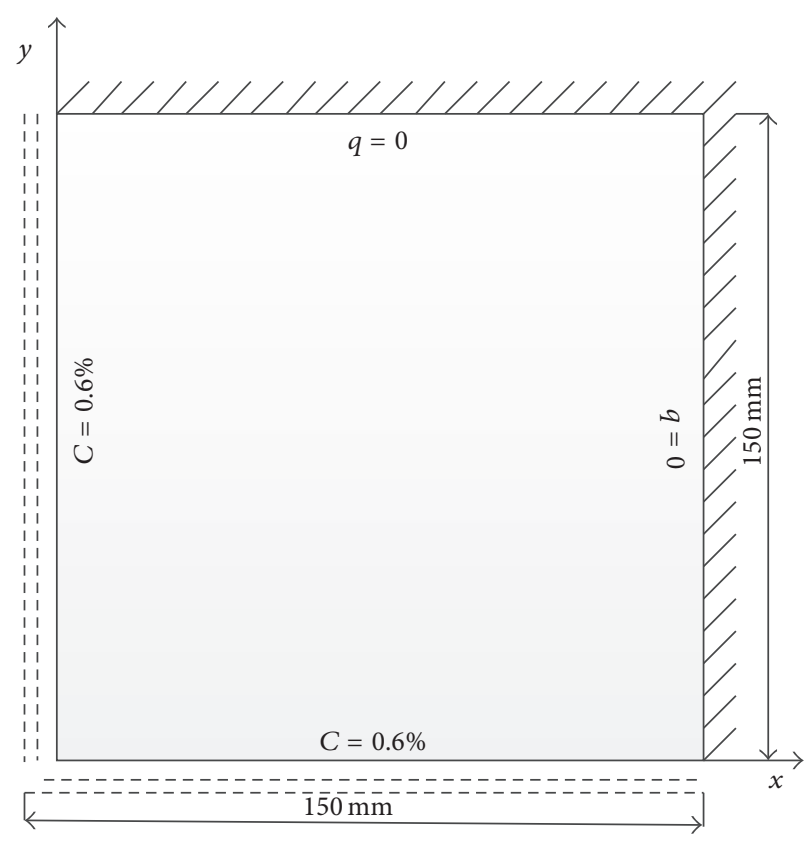

FIGURE 5: 2D chloride diffusion in a square concrete plate.

In the EFG method, $d_{\max }$ is an important scaling parameter; however only few studies have focused on the effects of its value. Moreover, the value of $d_{\max }$ to be chosen often depends on experience. The results obtained by the EFG method with four values of scaling parameter $\left(d_{\max }=1.01\right.$, $1.5,2.0,2.5$, and 3.0$)$ at the model location $(x=0.075)$ of the plate are shown in Table 5. Table 5 shows that the results obtained by $d_{\max }=(1.01,1.5,2.0$, and 2.5$)$ are similar and the maximum percentage $((A-B) / B) \times 100$ in the results obtained by the difference is less than $2 \%$, indicating that the scaling parameter $d_{\max }$ provided acceptable results for the range $1.0<d_{\max }<2.5$.

5.2.2. Coefficient D Is a Time-Dependent Function. In the following study the chloride diffusion coefficient $D$ is chosen as a time-dependent function and $m$ is 0.2 (depending on mix proportions and $0.1 \leq m \leq 1.2$ in [19]): $D=9.62 \times 10^{-12} \times$ $(0.1 / t)^{0.2} \mathrm{~m}^{2} / \mathrm{s}$. Other conditions are the same as those noted in the previous $2 \mathrm{D}$ problem. Figure 6 shows the calculated chloride concentration of the plate at $t=10$ years. Figure 6 shows that chloride concentrations at a depth of $60 \mathrm{~mm}$ for $t=10$ years are $0.35 \%, 0.28 \%$, and $0.27 \%$ according to MWLS, EFG, and FEM methods, respectively. These results show good agreement between the MWLS, EFG, and FEM methods. Table 6 shows the calculated initiation period when the start of rebar corrosion is set at $C_{t}=0.1 \%$ and the diagonal depth is $7.5 \mathrm{~cm}$. The CPU time of MWLS is 53 seconds and the CPU time of EFG is 95 seconds; numerical integration was no longer necessary for the MWLS method because the CPU time was less than that in the EFG method.

It can be seen that the initiation period of corrosion computed with MWLS is different from the results given by EFG and FEM when $D$ is a time-dependent function. Since MWLS is a weighted least square method, it does 
TABLE 4: Comparison of MWLS results with EFG, FEM, exact solutions at the specified locations $(x, y)$.

\begin{tabular}{llccrc}
\hline Location & & & Chloride concentration (\%), exposure time $t=5$ years \\
& & MWLS & EFG & $\begin{array}{c}\text { Exact } \\
\text { solutions }\end{array}$ \\
\hline \multirow{3}{*}{$x=0.075$} & $y=0.03$ & 0.4190 & 0.3926 & 0.3909 & 0.3909 \\
& $y=0.06$ & 0.2894 & 0.2363 & 0.2358 & 0.2355 \\
& $y=0.09$ & 0.2008 & 0.1501 & 0.1501 & 0.11497 \\
\hline
\end{tabular}

TABLE 5: Comparison of results with different $d_{\max }$ for chloride concentration at the location $(x=0.075)$.

\begin{tabular}{|c|c|c|c|c|c|c|c|}
\hline \multirow{3}{*}{ Location } & & \multicolumn{6}{|c|}{ Chloride concentration (\%), exposure time $t=5$ years } \\
\hline & & & & & $d_{\max }(A)$ & & \\
\hline & & $d_{\max }=1.01$ & $d_{\max }=1.5$ & $d_{\max }=2.0$ & $d_{\max }=2.5$ & $d_{\max }=3.0$ & Analytical solution $(B)$ \\
\hline$y=0.03$ & & 0.3926 & 0.3921 & 0.3922 & 0.3936 & 0.4157 & 0.3909 \\
\hline$y=0.06$ & $x-0075$ & 0.2363 & 0.2361 & 0.2361 & 0.2360 & 0.2402 & 0.2355 \\
\hline$y=0.09$ & $x=0.073$ & 0.1501 & 0.1520 & 0.1500 & 0.1498 & 0.1476 & 0.1497 \\
\hline$y=0.12$ & & 0.1151 & 0.1150 & 0.1150 & 0.1148 & 0.1108 & 0.1144 \\
\hline
\end{tabular}

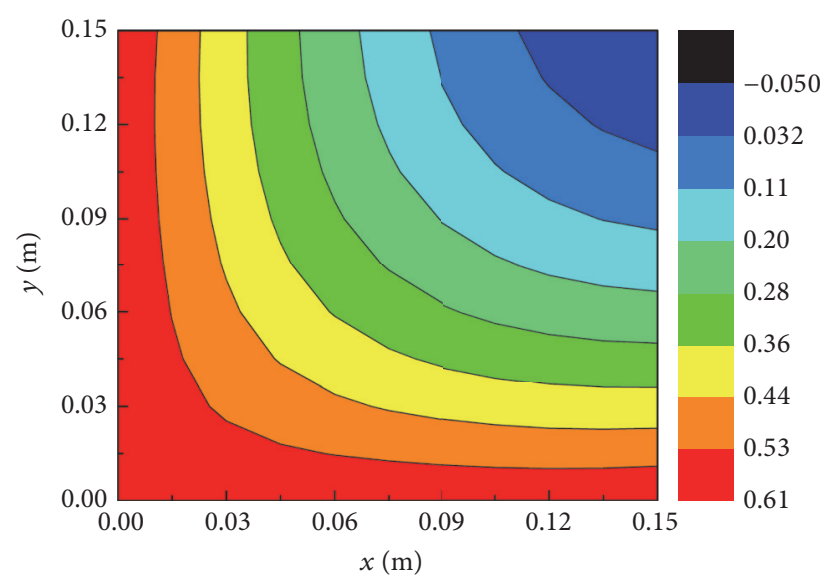

(a) MWLS

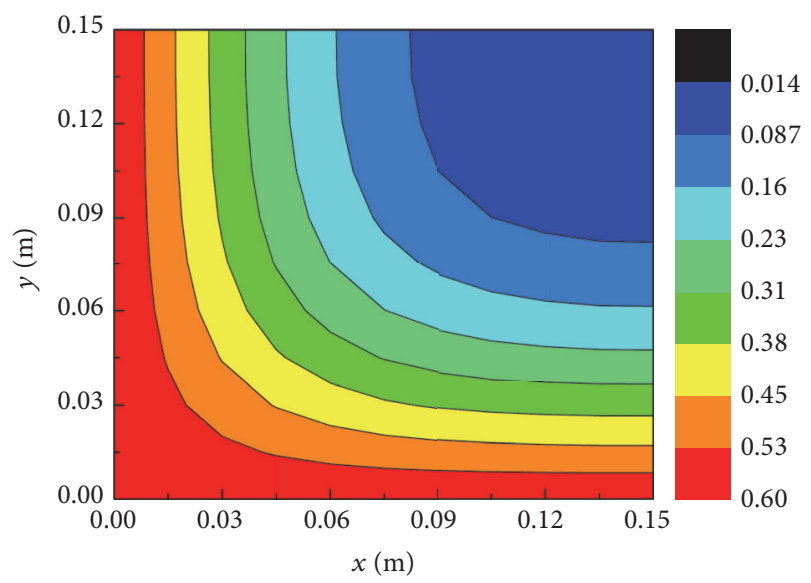

(b) EFG

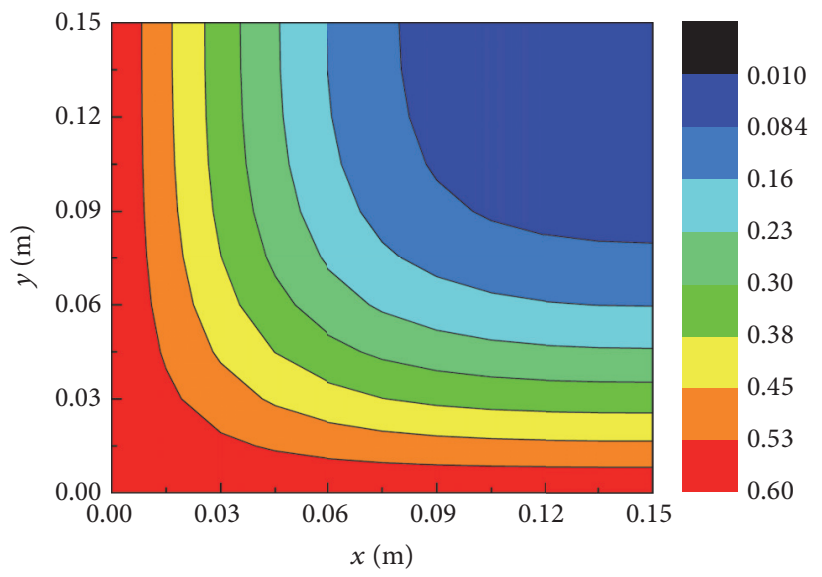

(c) FEM

FIGURE 6: Distribution of chloride concentration: (a) MWLS, (b) EFG, and (c) FEM at exposure time $t=10$ years. 


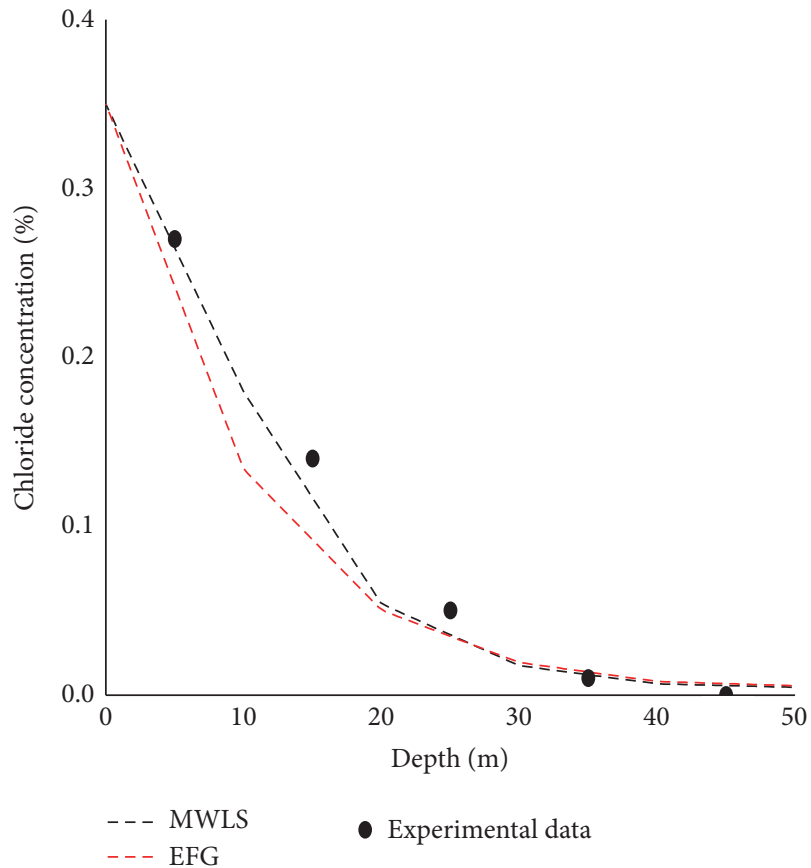

(a)

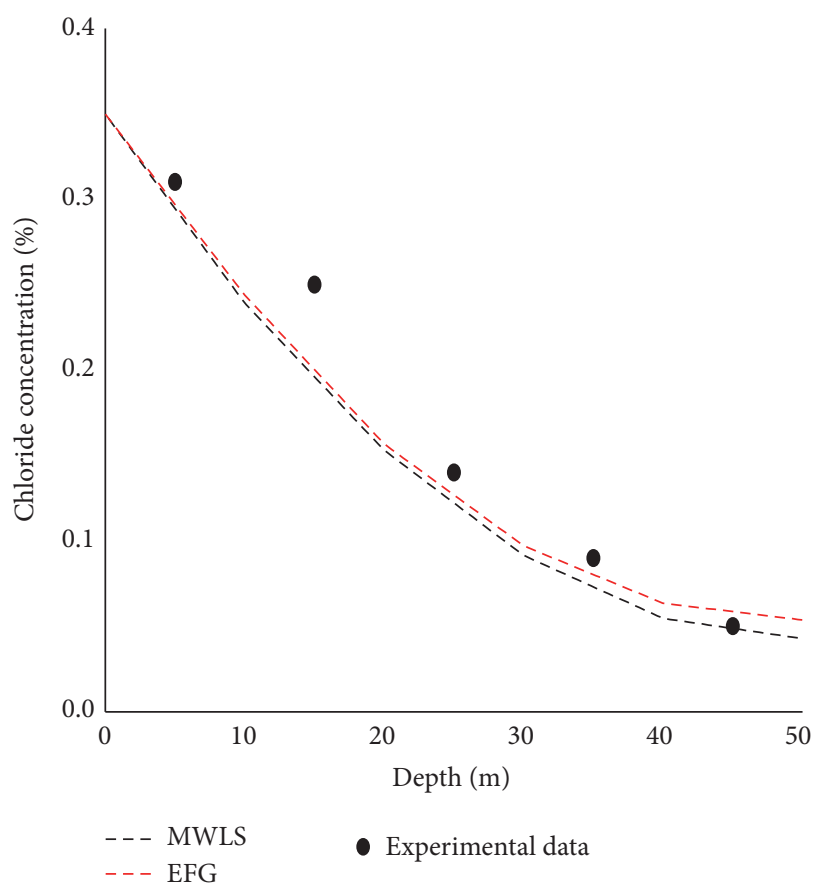

(b)

FIGURE 7: Comparison of chloride concentration computed by MWLS and EFG and experimentally measured data after exposure time: (a) $t=0.5$ years and $(\mathrm{b}) t=2$ years.

TABLE 6: The initiation period of corrosion as calculated by different methods.

\begin{tabular}{lcccc}
\hline $\begin{array}{l}\text { Initiation period } \\
\text { (year) }\end{array}$ & MWLS & EFG & FEM & Exact solutions \\
\hline $\begin{array}{l}D \text { is a constant } \\
\begin{array}{l}\text { is a time-dependent } \\
\text { function }\end{array}\end{array}$ & 3.00 & 3.15 & 3.30 & 3.25 \\
\hline
\end{tabular}

not entail numerical integration. Conversely, EFG and FEM methods need $n$-point Gauss quadrature. This explains the difference between MWLS results and those found by the other methods.

5.3. Chloride Diffusion in an Existing Marine Structure. An eight-year observation of chloride penetration in a real marine structure has been reported in [19]. Eighteen RC blocks were cast in 1987 and three mixes were considered. However, only the mix of Portland cement, $D=8 \times$ $10^{-12} \times(0.077 / t)^{0.1} \mathrm{~m}^{2} / \mathrm{s}$ and $C_{s}=0.35 \%$ is adopted in this paper. Figure 7 shows the chloride concentrations predicted by MWLS and EFG, as well as the measured data after exposure for two years and six months. Figure 7 shows that the numerical simulation aligned closely with experimental data.

These results still indicate the existence of an error between the numerical simulations and the experimental measurements. In the actual situation, the diffusion coefficient $D$ is not a constant and the chloride diffusion coefficient is a function both of time and depth and of temperature [18]. Chloride diffusion depends on the intrinsic permeability, which can be determined from the microporous structure of the concrete. However, the coefficient $D$ reported in [19] did not consider the effects of hydration and the microporous nature of concrete, and, hence, $D$ was only assumed to be a time-dependent function in this numerical model.

\section{Conclusions}

In this reported study, a MWLS method based on the weighted least squares approach and EFG method were used to address the problems in the simulation of chloride diffusion in concrete. A discrete function was adopted in the MWLS method, which avoided tedious numerical integration. A variety of $1 \mathrm{D}$ and $2 \mathrm{D}$ numerical examples demonstrated that the accuracy of MWLS is close to EFG, FEM, and an analytical solution. The EFG method provided an initiation period of corrosion in concrete that was close to FEM. However, the MWLS consumed much less computation time than EFG. Hence, the MWLS is an acceptable meshless method when computation time is considered, whereas the EFG is suitable when accuracy is considered. Both the MWLS and EFG methods successfully predicted the chloride concentration in concrete structure, which is used to prevent reinforcement corrosion in concrete structures.

\section{Competing Interests}

The authors declare that they have no competing interests. 


\section{References}

[1] M. K. Rahman, W. A. Al-Kutti, M. A. Shazali, and M. H. Baluch, "Simulation of chloride migration in compressioninduced damage in concrete," Journal of Materials in Civil Engineering, vol. 24, no. 7, pp. 789-796, 2012.

[2] X. Shi, N. Xie, K. Fortune, and J. Gong, "Durability of steel reinforced concrete in chloride environments: an overview," Construction and Building Materials, vol. 30, pp. 125-138, 2012.

[3] A. Limbeck, A. Eitzenberger, M. Bonta, and S. Burtscher, "New analysis method for the accurate determination of chloride content in the cement phase of concrete," in Proceedings of the 10th International Conference on Mechanics and Physics of Creep, Shrinkage, and Durability of Concrete and Concrete Structures (CONCREEP '15), pp. 800-804, Vienna, Austria, September 2015.

[4] C. Suwito and Y. Xi, "The effect of chloride-induced steel corrosion on service life of reinforced concrete structures," Structure and Infrastructure Engineering, vol. 4, no. 3, pp. 177-192, 2008.

[5] G. Sergi, S. W. Yu, and C. L. Page, "Diffusion of chloride and hydroxyl ions in cementitious materials exposed to a saline environment," Magazine of Concrete Research, vol. 44, no. 158, pp. 63-69, 1992.

[6] M. K. Kassir and M. Ghosn, "Chloride-induced corrosion of reinforced concrete bridge decks," Cement and Concrete Research, vol. 32, no. 1, pp. 139-143, 2002.

[7] T. Belytschko, Y. Y. Lu, and L. Gu, "Element-free Galerkin methods," International Journal for Numerical Methods in Engineering, vol. 37, no. 2, pp. 229-256, 1994.

[8] J. P. Boyd and K. W. Gildersleeve, "Numerical experiments on the condition number of the interpolation matrices for radial basis functions," Applied Numerical Mathematics, vol. 61, no. 4, pp. 443-459, 2011.

[9] X. Li, "Meshless Galerkin algorithms for boundary integral equations with moving least square approximations," Applied Numerical Mathematics. An IMACS Journal, vol. 61, no. 12, pp. 1237-1256, 2011.

[10] L. Yao, L. Zhang, X. Li, and L. Zhang, "Prediction of initiation time of corrosion in RC using meshless methods," Computers and Concrete, vol. 16, no. 5, pp. 669-682, 2015.

[11] X. Zhang, X.-H. Liu, K.-Z. Song, and M.-W. Lu, "Leastsquares collocation meshless method," International Journal for Numerical Methods in Engineering, vol. 51, no. 9, pp. 1089-1100, 2001.

[12] S. Abbasbandy and A. Shirzadi, "MLPG method for twodimensional diffusion equation with Neumann's and nonclassical boundary conditions," Applied Numerical Mathematics, vol. 61, no. 2, pp. 170-180, 2011.

[13] R. Salehi and M. Dehghan, "A moving least square reproducing polynomial meshless method," Applied Numerical Mathematics, vol. 69, pp. 34-58, 2013.

[14] J.-F. Wang and Y.-M. Cheng, "New complex variable meshless method for advection-diffusion problems," Chinese Physics B, vol. 22, no. 3, Article ID 030208, 2013.

[15] M. Bitaraf and S. Mohammadi, "Analysis of chloride diffusion in concrete structures for prediction of initiation time of corrosion using a new meshless approach," Construction and Building Materials, vol. 22, no. 4, pp. 546-556, 2008.

[16] L. Guo, T. Chen, and X.-W. Gao, "Transient meshless boundary element method for prediction of chloride diffusion in concrete with time dependent nonlinear coefficients," Engineering Analysis with Boundary Elements, vol. 36, no. 2, pp. 104-111, 2012.
[17] I. V. Singh, K. Sandeep, and R. Prakash, "Heat transfer analysis of two-dimensional fins using a meshless element free Galerkin method," Numerical Heat Transfer; Part A: Applications, vol. 44, no. 1, pp. 73-84, 2003.

[18] T. Luping and J. Gulikers, "On the mathematics of timedependent apparent chloride diffusion coefficient in concrete," Cement and Concrete Research, vol. 37, no. 4, pp. 589-595, 2007.

[19] M. D. A. Thomas and P. B. Bamforth, "Modelling chloride diffusion in concrete: effect of fly ash and slag," Cement and Concrete Research, vol. 29, no. 4, pp. 487-495, 1999. 

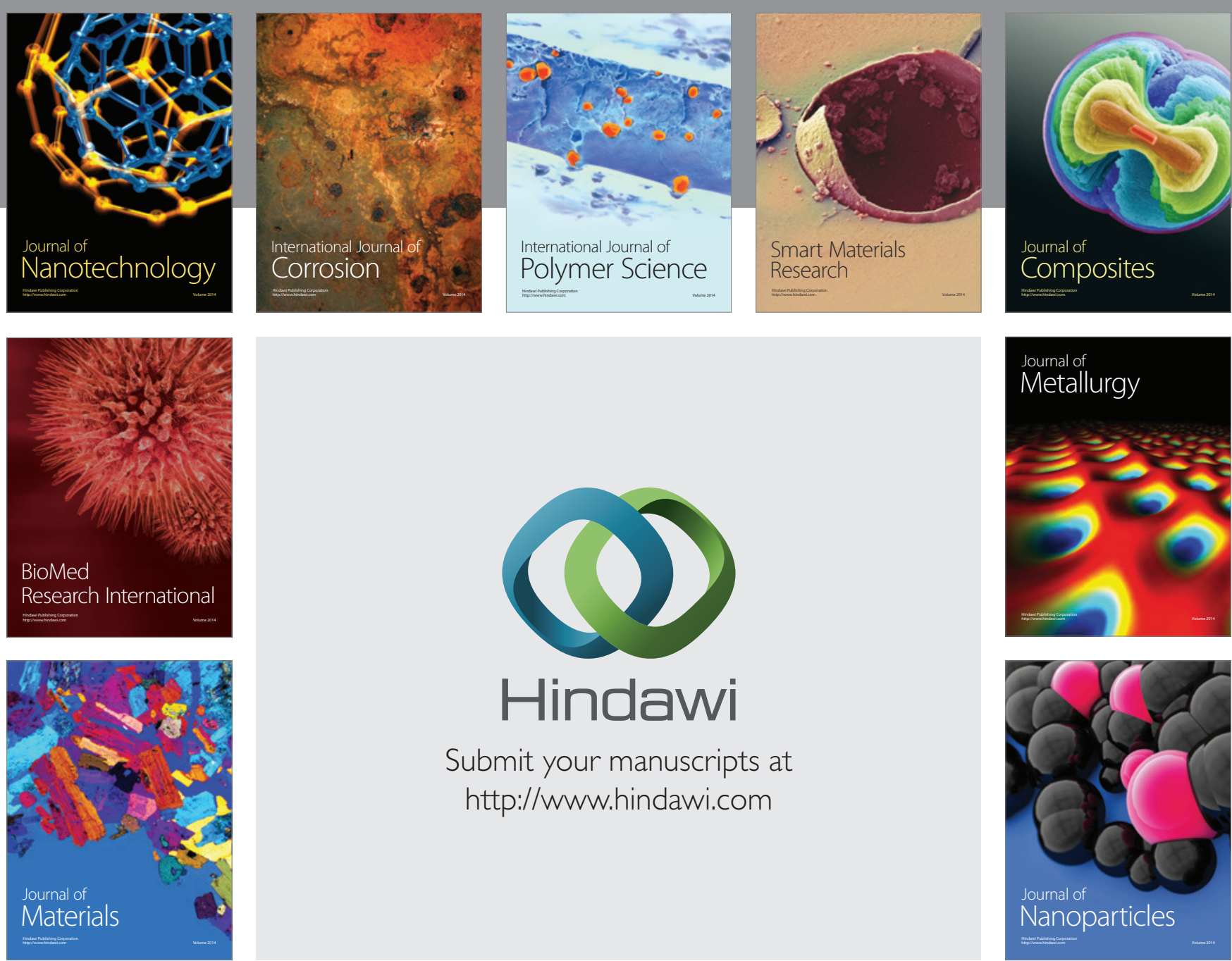

\section{Hindawi}

Submit your manuscripts at

http://www.hindawi.com

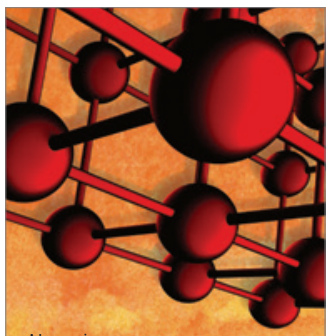

Materials Science and Engineering
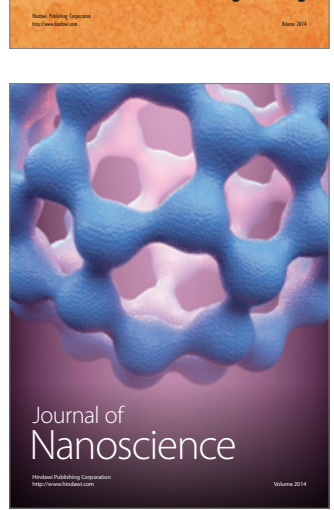
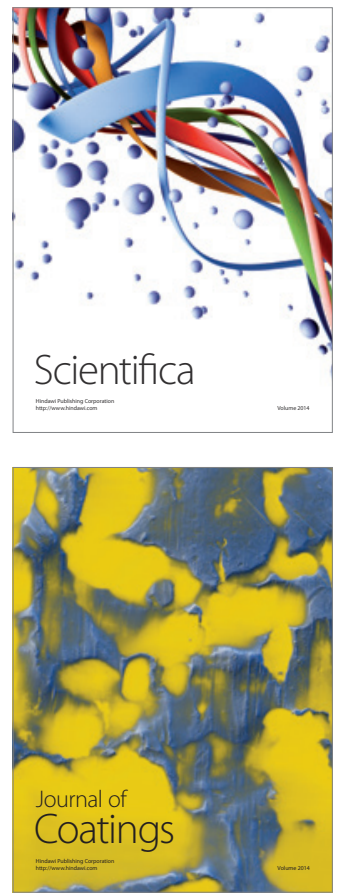
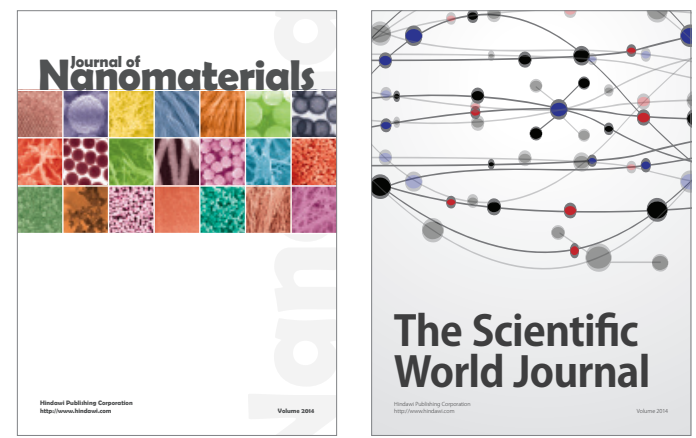

The Scientific World Journal
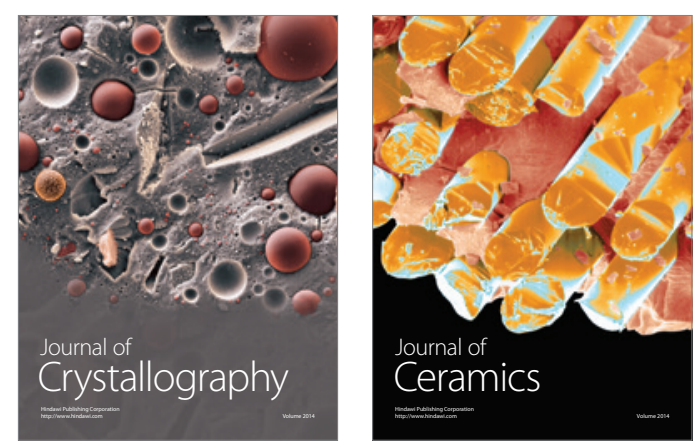
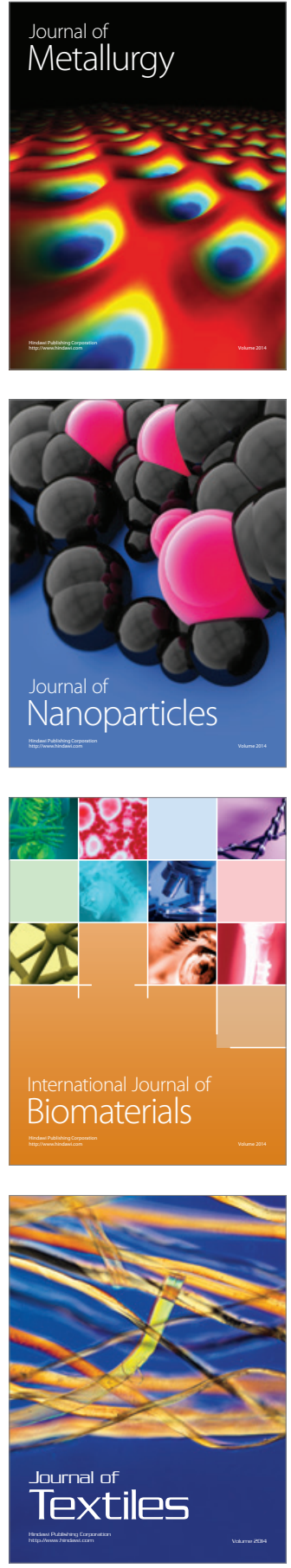